\title{
INCIDÊNCIA DE FÍSTULAS DIGESTIVAS NOS FERIMENTOS TORACOABDOMINAIS
}

\section{INCIDENCE OF DIGESTIVE FISTULA AFTER THORACOABDOMINAL WOUNDS}

\author{
Mario Mantovani, TCBC-SP 1 \\ Mauro José Pantoja Fontelles ${ }^{2}$ \\ Juliana Cristina Vieira de Freitas Resek Ajub ${ }^{3}$ \\ Flávia da Silva Pinto ${ }^{3}$
}

\begin{abstract}
RESUMO: Os ferimentos penetrantes localizados na transição toracoabdominal (FTA), além da dificuldade diagnóstica, merecem especial atenção em relação à conduta adotada para o controle das complicações abdominais. Os autores analisaram 110 pacientes atendidos na Disciplina de Cirurgia do Trauma do Departamento de Cirurgia da UNICAMP, de 1988 a 1998, apresentando ferida penetrante toracoabdominal e submetidos à laparotomia exploradora com drenagem pleural fechada. As fístulas digestivas foram estudadas quanto à incidência, ao tratamento e à evolução pós-operatória. Do total de pacientes, $91(82,7 \%)$ eram do sexo masculino e 19 $(17,3 \%)$ do sexo feminino. A faixa etária situou-se entre 13 e 63 anos. Os FTA foram causados por projétil de arma de fogo (PAF) em $60(54,5 \%)$ casos e por arma branca (FAB) em 50 (45,5\%). As fístulas digestivas ocorreram em seis $(5,4 \%)$ dos pacientes estudados, sendo quatro $(3,6 \%)$ casos de fístula pancreática, um $(0,9 \%)$ de fístula gástrica e um $(0,9 \%)$ de fístula biliar, todos tratados de maneira conservadora, apresentando evolução favorável com resolução espontânea.
\end{abstract}

Unitermos: Fístula digestiva, Ferimento toracoabdominal, Trauma torácico, Trauma abdominal, Trauma, Lesões do diafragma

\section{INTRODUÇÃO}

Estudos conduzidos na área do trauma têm dirigido especial atenção às lesões penetrantes localizadas em topografia a mais variada, em especial, àquelas que acometem a transição toracoabdominal com comprometimento do músculo diafragma-torácico. Estas, apresentam características próprias, sobretudo no que se refere à dificuldade diagnóstica e ao tratamento adequado. As lesões de vísceras ocas que, freqüentemente, acompanham estes ferimentos, podem ocasionar contaminação da cavidade peritonial com possibilidade de estender-se ao espaço pleural. ${ }^{1,2,3}$ Assim, nestes pacientes, não é rara a ocorrência de deiscência da sutura dos órgãos intra-abdominais atingidos, que leva ao aparecimento de fístulas digestivas, com graves repercussões hidreletrolíticas e nutricionais, responsáveis por elevadas taxas de morbidade e mortalidade. ${ }^{4,5}$

As controvérsias quanto ao tratamento são compreensíveis, dadas as dificuldades, já referidas, relativas ao diagnóstico correto. Por outro lado, como duas cavidades são atingidas, muito se discute sobre a conduta quanto às prioridades e às vias de acesso cirúrgico, com intuito de reduzir as possíveis complicações que podem acometer estas cavidades.

Tendo em vista estas dificuldades, foram revistos todos os casos de ferimentos toracoabdominais (FTA) ocorridos nos últimos dez anos, objetivando avaliar a incidência, o tratamento e a evolução das fístulas digestivas que acometeram estes pacientes.

\section{PACIENTES E MÉTODOS}

Em 1988, a disciplina de Cirurgia do Trauma da Universidade Estadual de Campinas - UNICAMP adotou um protocolo para tratamento das lesões produzidas por FTA. No presente trabalho, 110 pacientes foram selecionados, todos com ferimento toracoabdominal único e com indicação primária de laparotomia, sendo os casos cadastrados em um banco de dados utilizando-se o programa EPIINFO 6.04 (Center for Disease Control \& Prevention - CDC, USA).

Os FTA foram causados por projétil de arma de fogo (PAF) em $60(54,5 \%)$ casos e por arma branca (FAB) em 50

1. Professor Titular e Chefe da Disciplina de Cirurgia do Trauma do Departamento de Cirurgia da Faculdade de Ciências Médicas da UNICAMP.

2. Aluno do Curso de Pós-Graduação do Departamento de Cirurgia da Faculdade de Ciências Médicas da UNICAMP.

3. Acadêmica da Faculdade de Ciểncias Médicas da UNICAMP.

Recebido em 16/11/99

Aceito para publicação em 14/3/2000

Trabalho realizado pela Disciplina de Cirurgia do Trauma do Departamento de Cirurgia da Faculdade de Ciências Médicas da UNICAMP - Campinas-SP. 
(45,5\%), com o orifício de entrada localizado no tórax em $65(59,1 \%)$ pacientes e no abdome em $45(40.9 \%)$. Noventa e um $(82,7 \%)$ eram do sexo masculino e $19(17,3 \%)$ do sexo feminino. A faixa etária situou-se entre 13 e 63 anos. A distribuição da idade, por década, está representada na Tabela 1 .

Tabela 1

Número de casos conforme idade

\begin{tabular}{l|c|c|c}
\hline $\begin{array}{c}\text { Idade } \\
\text { (anos) }\end{array}$ & $\begin{array}{r}\text { No. de } \\
\text { casos }\end{array}$ & $\%$ & Acumulado \\
\hline $13-20$ & 26 & 23,7 & 23,7 \\
$21-30$ & 49 & 44,6 & 68,3 \\
$31-40$ & 23 & 20,9 & 89,2 \\
$41-50$ & 9 & 8,1 & 97,3 \\
$51-60$ & 2 & 1,8 & 99,1 \\
$61-63$ & 1 & 0,9 & 100,0 \\
\hline Total & 110 & 100,0 & \\
\hline
\end{tabular}

A determinação da pressão arterial sistólica, à admissão, revelou que em $95(86,3 \%)$ doentes os valores estavam acima de $80 \mathrm{mmHg}$, ao passo que, em $15(13,7 \%)$ esses valores eram iguais ou menores que $80 \mathrm{mmHg}$.

$\mathrm{O}$ atendimento inicial baseou-se nas prioridades de manutenção das vias aéreas com ventilação adequada e reposição volêmica. Estabilizadas as condições ventilatória e hemodinâmica, o paciente era encaminhado ao centro cirúrgico quando da indicação imediata de drenagem torácica e laparotomia. Exames complementares pertinentes ou observação clínica foram utilizados nos casos de dúvida quanto à penetração em cavidade. $\mathrm{O}$ tempo decorrido para o atendimento variou de um período de menos de duas a mais de dez horas. A maioria dos doentes $(85,4 \%)$ foi operada até quatro horas após a ocorrência do trauma (Tabela 2).

Todos foram submetidos à anestesia geral, com intubação orotraqueal, ventilação mecânica controlada e laparotomia mediana supra-umbilical, ampliada, quando necessário. Como orientação geral da disciplina de Cirur-

Tabela 2

Número de casos conforme o tempo decorrido da lesão até o atendimento

\begin{tabular}{c|c|c|c}
\hline $\begin{array}{c}\text { Tempo } \\
\text { (horas) }\end{array}$ & $\begin{array}{c}\text { No. } \\
\text { de casos }\end{array}$ & $\%$ & Acumulado \\
\hline$<2$ & 78 & 70,9 & 70,9 \\
$2-4$ & 16 & 14,5 & 85,4 \\
$4-6$ & 06 & 5,5 & 90,9 \\
$6-8$ & 02 & 1,8 & 92,7 \\
$8-10$ & 1 & 0,9 & 93,6 \\
$>10$ & 7 & 6,4 & 100,0 \\
\hline Total & 110 & 100,0 \\
\hline
\end{tabular}

gia do Trauma, os ferimentos de vísceras parenquimatosas que apresentavam sangramento ativo eram inicialmente tamponados com compressas e, após a melhora das condições hemodinâmicas do paciente, procediam-se às manobras cirúrgicas para controle eficiente da hemorragia. Durante a revisão sistemática das vísceras ocas, as perfurações encontradas eram ocluídas temporariamente com clamps ou suturas provisórias. Após evacuação dos coágulos e do sangue coletado, realizava-se a irrigação da cavidade com solução salina a $0,9 \%$; em seguida as vísceras atingidas eram tratadas de maneira definitiva. Pacientes com distúrbios da coagulação, acidose metabólica e hipotermia eram tratados com laparotomia abreviada (damage control) e posterior re-laparotomia assim que as condições clínicas permitissem.

A drenagem abdominal foi utilizada de maneira rotineira nos doentes que apresentavam ferimento hepático, pancreático, renal e do sistema excretor urinário e, no pósoperatório, os drenos abdominais foram progressivamente mobilizados até a retirada completa, variando o tempo de acordo com o volume, tipo de secreção e localização. Drenos próximos ao pâncreas e rins, eram mantidos por tempo mais prolongado em razão da possibilidade de formação de fístulas tardias.

Resíduos alimentares, secreções digestivas e fezes, mesmo constituindo-se em diferentes graus de contaminação, foram considerados como fatores contaminantes da cavidade peritonial (FCP). Trinta e três casos $(30,0 \%)$ apresentaram FCP. A relação dos órgãos atingidos, associada aos ferimentos diafragmáticos, está contida na Tabela 3.

Tabela 3

Número de casos conforme a víscera atingida*

\begin{tabular}{l|l|c|c}
\hline Tipo de víscera & Órgão & $\begin{array}{c}\text { No. } \\
\text { de casos }\end{array}$ & $\%$ \\
\hline Víscera oca & Estômago & 32 & 29,0 \\
& Cólon & 16 & 14,5 \\
& Delgado & 7 & 6,3 \\
& Duodeno & 4 & 3,6
\end{tabular}

Víscera parenquimatosa

\begin{tabular}{lll} 
Fígado & 63 & 57,2 \\
Baço & 34 & 30,9 \\
Rim & 19 & 17,2 \\
Pâncreas & 12 & 10,9 \\
\hline
\end{tabular}

* Vários pacientes com mais de um órgão atingido

Precedendo a laparotomia, o espaço pleural foi tratado com drenagem isolada em 107 casos, sendo que, oito deles $(7,3 \%)$ foram posteriormente submetidos à drenagem com irrigação e outros dois casos $(1,8 \%)$ à frenotomia com irrigação e drenagem. Além disso, três outros doentes $(2,7 \%)$ não foram submetidos à drenagem pleural isolada conjuntamente com a laparotomia.

Com base nos achados cirúrgicos, todos os doentes tiveram os índices de trauma calculados. $\mathrm{O}$ valor médio do 
RTS à admissão foi avaliado em 7.25 (0.0 - 7.84), o ISS com 22.3 (09 - 66) e o TRISS 0.90 (0.28 - 0.99). O valor do PATI (Penetrating Abdominal Trauma Index) ${ }^{6}$ no presente estudo, variou de 2 a 49, com média de 25.4 (Tabela 4).

Tabela 4

Valores relativos aos índices

\begin{tabular}{l|l|c}
\hline Índice & Variação & Valor médio \\
\hline RTS & $0 \quad-7,84$ & 7,25 \\
ISS & $9-66$ & 22,3 \\
TRISS & $0,28-0,99$ & 0,90 \\
PATI & $2-49$ & 25,4 \\
\hline
\end{tabular}

O diagnóstico das complicações baseou-se nos achados clínicos, laboratoriais e métodos de imagem (radiogramas).

Fístula ou deiscência de sutura gástrica ou intestinal foram diagnosticadas pelo tipo de secreção exteriorizada e pelo exame radiográfico (fistulografia).

A fistula pancreática foi identificada pela dosagem de amilase na secreção, em níveis superiores a 4.000 U.I. ou pelo achado operatório; ao passo que, a fistula biliar foi confirmada pela presença de bile no líquido drenado.

No presente estudo foram excluídos os pacientes com indicação de toracotomia ou toracofrenolaparotomia como via de acesso para correção de lesões torácicas e abdominais, assim como pacientes com evolução para óbito até o terceiro dia, inclusive, e pacientes com mais de um orifício de entrada no tronco.

\section{RESULTADOS}

Oitenta e seis $(78,2 \%)$ doentes evoluíram sem complicações abdominais no pós-operatório. A maioria $(74,5 \%)$ teve alta até o 10 o dia após a cirurgia (Tabela 5).

Tabela 5

Número de casos conforme a permanência hospitalar

\begin{tabular}{c|c|c}
\hline $\begin{array}{c}\text { Tempo de internação } \\
\text { (dias) }\end{array}$ & $\begin{array}{c}\text { No. } \\
\text { casos }\end{array}$ & $\%$ \\
\hline Até 10 & 82 & 74,5 \\
11 a 20 & 17 & 15,5 \\
20 a 30 & 06 & 5,5 \\
Após 30 & 05 & 4,5 \\
\hline Total & 110 & 100,0 \\
\hline
\end{tabular}

Fístulas digestivas - Foram observadas as seguintes: fístula pancreática, fístula biliar e fístula gástrica.

As fístulas digestivas ocorreram em seis $(5,4 \%)$ dos pacientes estudados, sendo quatro casos $(3,6 \%)$ de fístula pancreática, um $(0,9 \%)$ de fístula gástrica e um $(0,9 \%)$ de fístula biliar (Tabela 6).
Tabela 6

Número de casos conforme o tipo de fístula digestiva

\begin{tabular}{lc|c}
\hline Complicação abdominal & No. de casos & $\%$ \\
\hline Fístula pancreática & 4 & 3,6 \\
Fístula biliar & 1 & 0,9 \\
Fístula gástrica & 1 & 0,9 \\
\hline Total & 6 & 5,8 \\
\hline
\end{tabular}

Dois pacientes $(1,8 \%)$ faleceram após o terceiro dia de pós-operatório, por insuficiência de múltiplos órgãos e sistemas (IMOS).

\section{DISCUSSÃo}

A distribuição dos pacientes segundo idade e sexo, no presente trabalho, seguiu àquelas comumente encontradas nos centros de trauma urbanos. A média de idade de 27,4 \pm 9,6 anos, o predomínio do sexo masculino $(82,7 \%)$ e a maior incidência de lesões por projétil de arma de fogo $(54,5 \%)$ estão concordantes com a literatura compilada. ${ }^{2,}, 8,8$

Na avaliação pré-operatória, a condição hemodinâmica com pressão arterial sistólica acima de $80 \mathrm{mmHg}$ em $86,3 \%$ do pacientes retratou, adequadamente, a seleção feita na população dos casos com FTA. A exclusão dos doentes com lesões que exigiram a toracotomia ou toracofrenolaparotomia suprimiu a maior parte dos que apresentavam choque hemorrágico à admissão.

Excluindo-se os pacientes portadores de ferimentos torácicos com indicação de toracotomia para controle das lesões, a maioria dos autores é concordante que a laparotomia exploradora deva se constituir na via de acesso inicial no tratamento do FTA. $2,7,9,10$ Neste estudo, o local de penetração do agente vulnerante localizou-se no tórax em $59,1 \%$ dos casos, com a laparotomia como via de acesso único, independente da localização do orifício de entrada.

Não se dispõe, ainda, de meios seguros para diagnosticar a lesão do diafragma e vísceras abdominais nos pacientes sem sinais evidentes de irritação peritonial. A maioria das lesões diafragmáticas é descoberta durante a laparotomia para o tratamento das lesões associadas. Um alto índice de suposição é o fator mais importante no diagnóstico. ${ }^{9,} 10$ Trabalhos mais recentes, preconizam que o radiograma do tórax e a tomografia computadorizada não são métodos tão eficientes no diagnóstico da perfuração traumática do músculo diafragma como a videotoracoscopia assistida, que é, atualmente, preferível, por ser precisa, segura e minimamente invasiva, podendo, inclusive, servir como terapêutica para lesões intratorácicas. ${ }^{11,12}$

Ertekin et al. ${ }^{13}$ colocam a videolaparoscopia como método de importância crescente na detecção de lesões do diafragma em pacientes sem outras indicações para laparotomia.

Não raro, passam despercebidas lesões diafragmáticas nos ferimentos por arma branca na transição toracoabdominal. A suspeita aliada à utilização da videotoracoscopia ou videolaparoscopia são os métodos por nós utilizados. Quando o ferimento é à direita do diafragma, 
nossa preferência é pela videotoracoscopia que, quando positiva, indica uma videolaparoscopia ou laparotomia. Nos ferimentos à esquerda do diafragma, preferimos a videolaparoscopia que pode ser diagnóstica e/ou terapêutica. No presente trabalho, utilizamos o método nos últimos 30 casos.

Neste estudo, a drenagem sob selo d'água, associada à laparotomia, foi suficiente para tratar o espaço pleural em $88,2 \%$ dos pacientes. Os autores consultados referem-se à presença de restos alimentares e/ou fezes na cavidade como um dos critérios para escolha da conduta no tratamento do espaço pleural. ${ }^{1,2}$ A persistência de comunicação entre as duas cavidades pode dar origem a complicações, tais como: o empiema pleural, fístulas pleurobiliares e fístulas gastropleurais, comunicando-se ou não com as vias respiratórias. ${ }^{7}$ Neste sentido, no músculo diafragma-torácico, por ser a estrutura anatômica cuja lesão determina o diagnóstico de FTA, a ferida de qualquer extensão deve sempre ser reparada, com intuito de evitar aspiração de secreção do espaço subfrênico e conseqüente risco de herniação de vísceras abdominais para o espaço pleural. Isto ocorre em razão do gradiente pleuroperitonial que, durante a respiração normal, está entre 7 e $20 \mathrm{cmH}_{2} \mathrm{O}$, podendo chegar a valores de $100 \mathrm{cmH}_{2} \mathrm{O}$ durante a inspiração profunda. ${ }^{2,7,14}$

Nesta casuística, hemidiafragma esquerdo foi o mais afetado, estando presente em $75(68,1 \%)$ dos pacientes estudados, o que está de acordo com a literatura. ${ }^{9,10}$

Ao correlacionar-se o PATI, no presente trabalho, com as complicações pós-operatórias, constatou-se que estas estiveram associadas com altos valores do índice. Moore et al. ${ }^{6}$, observaram que índices maiores que 25 estavam associados a taxas de complicações de $46 \%$ e $50 \%$, respectivamente, para lesões por arma branca e projétil de arma de fogo. Estes dados contrastam com os valores observado por Bernini ${ }^{2}$, que não observou aplicabilidade do PATI em sua casuística. Os valores médios do RTS $(7,25)$ e ISS (22,3), calculados à admissão, estiveram concordantes com as lesões encontradas; o valor médio do TRISS $(0,90)$ ficou abaixo do índice de sobrevivência encontrado nos pacientes que evoluíram sem óbito $(98,2 \%)$.

A freqüência com que os órgãos abdominais foram atingidos não foi diferente da referida por outros autores, com exceção do baço e do estômago. 1,2, 5, 15, 16, 17 O fígado, ocupando área correspondente ao hemidiafragma direito e parte do esquerdo, foi a víscera abdominal mais atingida, com $63(57,3 \%)$ pacientes, seguido pelo baço com 34 $(30,9 \%)$, estômago $32(29,1 \%)$, rim $19(17,3 \%)$ e cólon com $16(14,5 \%)$ casos.

Quanto ao agente vulnerante, os ferimentos por projétil de arma de fogo, que acometem duas ou mais vísceras em $95 \%$ dos casos, estiveram mais associados às complicações abdominais. Isto se justifica pela maior lesão tecidual produzida por este tipo de agente, o que é menos freqüente nas lesões produzidas por arma branca.

O uso de antimicrobianos seguiu a padronização do Serviço da Disciplina de Cirurgia do Trauma. Cefalosporina de primeira geração foi administrada em $64(58,2 \%)$ pacientes, seguida em freqüência pela sua associação com metronidazol e aminoglicosídeos. O uso de antibióticos nos ferimentos abdominais penetrantes não desperta controvérsias, pois a indicação é quase sempre terapêutica, em virtude da freqüente contaminação da cavidade peritonial por bactérias da flora intestinal. Relatos da literatura alertam para o efeito benéfico dos antibióticos em reduzir a incidência de complicações infecciosas nestes pacientes. Apesar do desenvolvimento de novas drogas e suas associações, a sépsis continua como principal causa de morbidade e mortalidade tardias no trauma abdominal. ${ }^{18,19}$

Outro fator que pode influenciar a evolução pós-operatória é a drenagem da cavidade abdominal. Em razão do acometimento mais freqüente das vísceras do andar supramesocólico e da dinâmica da distribuição dos líquidos intraperitoniais, a tendência é acumularem-se as secreções nos espaços subfrênicos. Fatores como: as deiscências de suturas, o extravasamento de conteúdo gastrointestinal e a contaminação de coágulos, podem favorecer o acúmulo destas secreções. As lesões traumáticas ou cirúrgicas do fígado, pâncreas e rins, exigem a drenagem e a permanência do dreno até que se tenha certeza que não houve necrose, liquefação ou fistulização.

As lesões de estômago tornam-se mais importantes à medida que o empiema pleural é a complicação mais freqüentemente associada à lesão deste órgão, quando o diafragma está comprometido. A incidência de empiema em tais casos é de três a quatro vezes mais alta do que em trauma penetrante isolado do tórax. ${ }^{15}$

As fístulas digestivas são complicações pouco freqüentes no trauma abdominal, ocorrendo como conseqüência da deiscência de suturas das vísceras atingidas, o que se verifica por volta do sétimo dia de pós-operatório. A mortalidade relatada com este tipo de complicação varia entre $6,5 \%$ e $21 \%$, sempre associada à grande perda de líquidos, com distúrbios hidreletrolíticos, desnutrição e sépsis. ${ }^{16,17}$ Os valores encontrados, no presente trabalho $(5,8 \%)$, estão concordantes com os relatos da literatura. ${ }^{16}$, $17,20,21$

As fístulas gástricas são iatrogênicas em $70 \%$ a $90 \%$ dos casos e, freqüentemente, ocorrem após procedimentos cirúrgicos sobre o estômago, duodeno ou trato biliar. O trauma é causa pouco comum deste tipo de fístula, incidindo em $0,6 \%$ dos pacientes ${ }^{16}$, estando, portanto, concordante com esta casuística $(0,9 \%)$.

A fístula pancreática pode ser interna ou externa, sendo conseqüência de lesão do ducto pancreático ou pancreatite. É a complicação mais comum resultante de lesão traumática sobre o pâncreas ou duodeno e, em $70 \%$ dos casos, evolui para cura espontaneamente, em especial, quando o débito é baixo. ${ }^{16,20} \mathrm{~A}$ evolução deste tipo de complicação foi bem evidenciada em trabalho de Birolini et al. ${ }^{20}$, em que foi constatada sua resolução espontânea, embora às custas de longo período de internação e vigilância clínica constante. Neste trabalho esteve presente em quatro $(3,6 \%)$ dos pacientes estudados.

A fístula biliar pós-traumática ocorre por lesão com comprometimento da árvore biliar intra ou extra-hepática. A contusão profunda do abdome é a causa mais comum e a que determina lesões mais graves, tanto do fígado como de outros órgãos. Agudelo ${ }^{21}$, relata uma incidência de $0,6 \%$ de fistula biliar em pacientes com traumatismo hepático submetidos à laparotomia. No presente estudo, 
esta complicação apresentou evolução benigna, incidindo em um $(0,9 \%)$ dos pacientes e esteve relacionada com a víscera mais atingida: o fígado.

Nesta casuística, todos os casos que se complicaram com fístula digestiva no pós-operatório foram tratados de maneira conservadora, apresentando evolução favorável com resolução espontânea.
Assim, deve-se ter sempre em mente que, as fístulas representam complicações associadas a diversas afecções do trato digestivo, incluindo as intervenções cirúrgicas para correção das lesões decorrentes dos ferimentos toracoabdominais, podendo cursar com significantes morbidade e mortalidade resultantes de grande perda de líquidos com desequilíbrio hidreletrolítico, desnutrição ou sépsis.

\begin{abstract}
Penetrating trauma in the lower chest, is a diagnosis challenge and deserves special attention with respect to the treatment for abdominal complications. One hundred and ten patients admitted to the emergency unit of UNICAMP - University Hospital Trauma Center from 1988 to 1998 who presented thoracoabdominal penetrating wounds and were submitted to laparotomy and closed tube thoracostomy were analysed. Incidence, treatment and postoperative evolution. Digestive fistula was studied. Ninety-one patients (82,7\%) were male and $19(17,3 \%)$ female, with the age varying between 13 to 63 years old. Gunshot wounds ocurred in $60(54,5 \%)$ patients, while stab wounds in 50 (45,5\%). Digestive fistula occurred in six (5,4\%) patients; four (3,6\%) were pancreatic fistula, one $(0,9 \%)$ biliar which and one $(0,9 \%)$ gastric were managed nonoperative with a satisfactory evolution.
\end{abstract}

Keywords: Digestive fistula, Chest wounds, Thoracoabdominal wounds, Abdominal trauma, Diaphragmatic injuries, Trauma

\section{REFERÊNCIAS}

1. Borja AR, Ransdell HT: Treatment of thoraco-abdominal gunshot wounds in civilian pratice. Am J Surg 1971; 121:580-2.

2. Bernini CO: Ferimentos toracoabdominais: estudo de fatores determinantes de complicações pleuropulmonares. Tese de Doutorado, 1992. Faculdade de Medicina - USP.

3. Wylie RH, Hoffmann HL, Williams DB, et al.: The thoracoabdominal casuality. Ann Surg 1946; 124:46378.

4. Adkins RR, Woltering EA: Penetrating chest wall and thoracic injuries. Am J Surg 1985; 51:104-8.

5. Oparah SS, Mandal AK: Penetrating gunshot wounds of chest in civilian pratice: experience with 250 consecutives cases. Br J Surg 1978; 65:45-8.

6. Moore EE, Dunn EL, Moore JB, et al.: Penetrating abdominal trauma index. J Trauma 1981; 21:439-45.

7. Miller L, Bennett EV, Root HD, et al: Management of penetrating and blunt diaphragmatic injury. J Trauma 1984; 24:403-9.

8. Mandal AK, Thadepalli H, Mandal AK, et al: Posttraumatic Empiema Thoracis: A 24 Year Experience at a Major Trauma Center. J Trauma 1997; EUA, 5:764 - 71.

9. Demetriades D, Kakoyannis S, Parekh D, et al: Penetrating injuries of the diaphragm. Br J Surg 1988; 75:824-6.

10. Adamthwaite DN: Penetrating injuries of the diaphragm. Injury $1981 ; 14: 151-8$

11. Smith S, Fry WR, Tsoi EK, et al: Preliminary report on videothoracoscopy in the evaluation and treatment of thoracic injury. Am J Surg 1993; 166:690-3.

12. Span JC, Nwariaku FE, Wait M: Evaluation of video-assisted thoracoscopic surgery in the diagnosis of diaphragmatic injuries. Am J Surg 1995; 170:628-31.
13. Ertekin C, Onaran Y, Guloglu R, et al: The use of laparoscopy as a primary diagnostic and therapeutic methodo in penetrating wounds of lower thoracal region. Ser Laparosc Endosc 1998; 8:26-9.

14. Pipikin NL, Hamit HF: Traumatic perfuration of the diaphragm. South Med 1988; 81:1347-50.

15. Wiencek RG, Wilson RF, Steiger Z: Acute injuries of diaphragm. J Thorac Cardiovac Surg 1986; 92:988-93.

16. Durham RM, Olson S Wiejelt JA: Penetrating injuries to the stomach. Surg Gynecol Obstet 1991; 172:298-302.

17. Foster III CE \& Lefort AT: General management of gastrointestinal fistulas. Clin Srg North Am 1996; 76:101933.

18. Flynn NM, Lawrence RM: Antimicrobial prophylaxis. Med Clin North Am 1979; 63:1225-44.

19. Hofstetter SR, Pachter HL, Bailey AA, et al.: A prospective comparison of two regimens of prophylatic antibiotics in abdominal trauma: cefoxitin versus triple drug. J Trauma 1984; 24:307-10.

20. Birolini D, Coloma HG, Bevilacqua RG, et al: Fístulas pancreáticas externas pós-traumáticas. AMB Rev Assoc Med Bras 1985; 31:20-4.

21. Agudelo CL: Trauma hepático durante un periodo de 10 anos: analisis de 1675 casos. Med. UPB 1986; 5:1725 .

\section{ENDEREÇO PARA CORRESPONDÊNCIA}

Dr. Mario Mantovani

Rua José de Campos Salles, 650 - Jardim Paraíso

13095-300 - Campinas-SP 